\title{
Introduction to Neutrosophic Topological Spatial Region, Possible Application to GIS Topological Rules
}

\author{
A. A. Salama ${ }^{1}$, Said Broumi ${ }^{2}$, S. A. Alblowi ${ }^{3}$ \\ ${ }^{1}$ Department of Mathematics and Computer Science, Faculty of Sciences, Port Said University, 23 December Street, Port \\ Said 522, Egypt. \\ ${ }^{2}$ Faculty of Lettres and Humanities, Hay El Baraka Ben M'sik Casablanca B.P. 7951 University of Hassan II, casablanca \\ Morocco. \\ ${ }^{3}$ Department of Mathematics, King Abdulaziz University, Jeddah, Saudi Arabia \\ Email: drsalama44@gmail.com, broumisaid78@gmail.com, salwaalblowi@ hotmail.com
}

\begin{abstract}
Neutrosophic set is a power general formal framework, which generalizes the concept of the classic set, fuzzy set, interval valued fuzzy set, intuitionistic fuzzy set, and interval intuitionistic fuzzy set from philosophical point of view. In Geographical Information Systems (GIS) there is a need to model spatial regions with indeterminate boundary and under indeterminacy. In this paper, we first gives fundamental concepts and properties of a neutrosophic spatial region.
\end{abstract}

Index Terms-Neutrosophic Sets, Neutrosophic Topology, Geographical Information Systems, Neutrosophic Spatial Region.

\section{INTRODUCTION}

Since the world is full of indeterminacy, the neutrosophics found their place into contemporary research. The paper will discuss several possible contributions to the GIS field including remote sensing, object reconstruction from airborne laser scanner, real time tracking, routing applications and modeling cognitive agents. In this paper, a simple neutrosophic region and fundamental concepts for uncertainty and indeterminacy modeling of spatial relationships are analyzed from the viewpoint of neutrosophic (NS) logic. This paper gives fundamental concepts and properties of a neutrosophic spatial region.

\section{RELATED WORK}

Algebraic topological models for spatial objects were introduced in (White 1979). Thirteen topological relations between two temporal intervals were identified by J.F.Allen [6]. After the 4-intersection model proposed by M.J Egenhofer [16], M.J. Egenhofer and R.D.Franzosa [17], the 9-intersection approach introduced by M.J Egenhofer and J. R. Herring [18] was proposed as a formalism for topological relations. This approach is based on point-set topological concepts. In the 9-intersection method, a spatial object $\mathrm{A}$ is decomposed into three parts: an interior denoted by Ao, an exterior denoted by $\mathrm{AE}$, and a boundary denoted by $\partial \mathrm{A}$. There are nine intersections between six parts of two objects. The other significant approach known as RCC (Region-Connection Calculus) has been provided by Cohn et al. D.A. Randell, Z. Cui [25]; M.N.Gotts, J.M.Gooday et al. [21]; A.G.Cohn, B.Bennet et al. [12]. During recent years, the topological relations have been extended into fuzzy domains. An example of a fuzzy object was provided by P. Fisher [19]. A number of papers of M. Schneider [27, 28, 29, 30] was presented to model fuzzy set in GIS community and to design a system of fuzzy spatial data types including operations and predicates. M. Molenaar [24] extended the formal model into fuzzy domain and based on this model $\mathrm{T}$. Cheng [8] proposed a process-oriented spatio-temporal data model. The intersection model is extended to vague regions by three main approaches: the work of $\mathrm{E}$. Clementini and Di Felice $[9,11]$ on regions with "broad boundary", the work of F. B Zhan [34] who developed a method for approximately analyzing binary topological relations between geographic regions with indeterminate boundaries based on fuzzy sets, and X. Tang and W. Kainz [32] that provided a $3 * 3$, a $4 * 4$, and a $5 * 5$ intersection matrix based on different topological parts of two fuzzy regions. The extension of the RCC schemes to accommodate vague region has been ad-dressed by F.Lehmann and A.G Cohn [23], and by A.G.Cohn and M.N .Gotts [13]. In this direction J.G. Stell and M.F Worboys [31] have used Heyting structures. The notion of intuitionistic ets (IFS) was introduced by K. Atanassov. $[1,2,3]$ as generalization of fuzzy sets. The notion of neutrosophic sets (NS) was introduced by F. Smarandache [45, 46, 47] as generalization of intuitionistic fuzzy sets. In other research, A. A Salama [36] introduced the concept of neutrosophic topology. A. A. Salama, et,al [42] proposed a new mathematical model called "Neutrosophic crisp Sets and Neutrosophic crisp Topological Spaces.

\section{PRELIMINARIES}


First we present the fundamental concepts and definitions given by Salama and Smarandache. We recollect some relevant basic preliminaries, and in particular, the work of Smarandache in [7, 8], Atanassov in [1, 2, 3] and Salama [9]. Smarandache introduced the neutrosophic components T, I, F which represent the membership, indeterminacy, and non-membership values respectively, where $] 0^{-}, 1^{+}[$is nonstandard unit interval.

\section{Definition 3.1[47]}

Let T, I, F be real standard or nonstandard subsets of $\left|0^{-}, 1^{+}\right|$, with

$$
\begin{aligned}
& \text { Sup_T=t_sup, inf_T=t_inf } \\
& \text { Sup_I=i_sup, inf_I }=\mathrm{i} \text { inf } \\
& \text { Sup_F=f_sup, inf_F=f_inf } \\
& \text { n-sup=t_sup+i_sup+f_sup } \\
& \text { n-inf=t_inf+i_inf+f_inf, }
\end{aligned}
$$

T, I, F are called neutrosophic components

We shall now consider some possible definitions for basic concepts of the neutrosophic set and its operations due to Salama et al [ ].

\section{Definition 3.2}

Let $\mathrm{X}$ be a non-empty fixed set. A neutrosophic set ( NS for short) $\mathrm{A}$ is an object having the form $A=\left\{\left\langle x, \mu_{A}(x), \sigma_{A}(x), \gamma_{A}(x)\right\rangle: x \in X\right\} \quad$ where $\mu_{A}(x), \sigma_{A}(x)$ and $\gamma_{A}(x)$ which represent the degree of member ship function (namely $\mu_{A}(x)$ ), the degree of indeterminacy (namely $\sigma_{A}(x)$ ), and the degree of non-member ship (namely $\left.\gamma_{A}(x)\right)$ respectively of each element $x \in X$ to the set $\mathrm{A}$.

\section{Remark 3.1}

A neutrosophic $A=\left\{<x, \mu_{A}(x), \sigma_{A}(x), \gamma_{A}(x)>: x \in X\right\}$ can be identified to an ordered triple $\left\langle\mu_{A}, \sigma_{A}, \gamma_{A}\right\rangle$ in ]$^{-} 0,1^{+}[$on $\mathrm{X}$.

\section{Remark 3.2}

For the sake of simplicity, we shall use the symbol $A=<x, \mu_{A}, \sigma_{A}, \gamma_{A}>$ for the NS $A=\left\{<x, \mu_{A}(x), \sigma_{A}(x), \gamma_{A}(x)>: x \in X\right\}$

\section{Example 3.1}

Every IFS A a non-empty set $\mathrm{X}$ is obviously on $N S$ having the form $A=\left\{<x, \mu_{A}(x), 1-\left(\mu_{A}(x)+\gamma_{A}(x)\right), \gamma_{A}(x)>: x \in X\right\}$

Since our main purpose is to construct the tools for developing neutrosophic set and neutrosophic topology, we must introduce the NSS $0_{N}$ and $1_{N}$ in $X$ as follows:

$0_{N}$ may be defined as four types:

$\left(0_{1}\right)$ Type 1. $0_{N}=\{\langle x, 0,0,1\rangle: x \in X\}$

$\left(0_{2}\right)$ Type 2. $0_{N}=\{\langle x, 0,1,1\rangle: x \in X\}$ $\left(0_{3}\right)$ Type 3. $0_{N}=\{\langle x, 0,1,0\rangle: x \in X\}$

$\left(0_{4}\right)$ Type 4. $0_{N}=\{\langle x, 0,0,0\rangle: x \in X\}$

$1_{N}$ may be defined as four types:

(1, Type 1. $1_{N}=\{\langle x, 1,0,0\rangle: x \in X\}$

$\left(1_{2}\right)$ Type 2. $1_{N}=\{\langle x, 1,0,1\rangle: x \in X\}$

$\left(1_{3}\right)$ Type $31_{N}=\{\langle x, 1,1,0\rangle: x \in X\}$

$\left(1_{4}\right)$ Type 4. $1_{N}=\{\langle x, 1,1,1\rangle: x \in X\}$

\section{Definition 3.3}

Let $A=\left\langle\mu_{A}, \sigma_{A}, \gamma_{A}\right\rangle$ a $N S$ on $X$, then the complement of the set $A(C(A)$, for short ) maybe defined as three kinds of complements
$\left(C_{1}\right) C(A)=\left\{\left\langle x, 1-\mu_{A}(x), 1-\gamma_{A}(x)\right\rangle: x \in X\right\}$,
$\left(C_{2}\right) C(A)=\left\{\left\langle x, \gamma_{A}, \sigma_{A}(x), \mu_{A}(x)\right\rangle: x \in X\right\}$
$\left(C_{3}\right) \quad C(A)=\left\{\left\langle x, \gamma_{A}, 1-\sigma_{A}(x), \mu_{A}(x)\right\rangle: x \in X\right\}$

One can define several relations and operations between NSS follows:

\section{Definition 3.4}

Let $\mathrm{X}$ be a non-empty set, and NSS A and B in the form $A=\left\langle x, \mu_{A}(x), \sigma_{A}(x), \gamma_{A}(x)\right\rangle, B=\left\langle x, \mu_{B}(x), \sigma_{B}(x), \gamma_{B}(x)\right\rangle$, then we may consider two possible definitions for subsets $(A \subseteq B)$

$(A \subseteq B)$ may be defined as two types:

(1) Type 1: $A \subseteq B \Leftrightarrow \mu_{A}(x) \leq \mu_{B}(x), \gamma_{A}(x) \geq \gamma$ and $\sigma_{A}(x) \leq \sigma_{B}(x) \quad \forall x \in X$

(2) Type 2: $A \subseteq B \Leftrightarrow \mu_{A}(x) \leq \mu_{B}(x), \gamma_{A}(x) \geq \gamma_{B}(x)$ and $\sigma_{A}(x) \geq \sigma_{B}(x)$

\section{Proposition 3.1}

For any neutrosophic set $A$ the following are holds

(1) $0_{N} \subseteq A, \quad 0_{N} \subseteq 0_{N}$

(2) $A \subseteq 1_{N}, 1_{N} \subseteq 1_{N}$

\section{Definition 3.5}

Let $\mathrm{X}$ be a non-empty set, and

$$
\begin{aligned}
& A=<x, \mu_{A}(x), \gamma_{A}(x), \sigma_{A}(x)>, \\
& B=<x, \mu_{B}(x), \sigma_{B}(x), \gamma_{B}(x)>\text { are NSS. Then }
\end{aligned}
$$

(1) $A \cap B$ may be defined as three types: 
$\left(I_{1}\right)$ Type1: $A \cap B=<x, \mu_{A}(x) \cdot \mu_{B}(x), \sigma_{A}(x) \cdot \sigma_{B}(x)$, $\gamma_{A}(x) \cdot \gamma_{B}(x)>$

$\left(I_{2}\right)$ Type 2: $A \cap B=<x, \mu_{A}(x) \wedge \mu_{B}(x), \sigma_{A}(x) \wedge \sigma_{B}(x)$, $\gamma_{A}(x) \vee \gamma_{B}(x)>$

$\left(I_{3}\right)$ Type 3: $A \cap B=<x, \mu_{A}(x) \wedge \mu_{B}(x), \sigma_{A}(x) \vee \sigma_{B}(x)$, $\gamma_{A}(x) \vee \gamma_{B}(x)>$

(2) $A \cup B$ may be defined as two types :

$\left(U_{1}\right)$ Type1: $A \cup B=<x, \mu_{A}(x) \vee \mu_{B}(x), \sigma_{A}(x) \vee \sigma_{B}(x)$, $\gamma_{A}(x) \wedge \gamma_{B}(x)>$

$\left(U_{2}\right)$ Type 2:

$A \cup B=<x, \mu_{A}(x) \vee \mu_{B}(x), \sigma_{A}(x) \wedge \sigma_{B}(x)$,

$\gamma_{A}(x) \wedge \gamma_{B}(x)>$

(3) []$A=<x, \mu_{A}(x), \sigma_{A}(x), 1-\mu_{A}(x)>$ $<>A=<x, 1-\gamma_{A}(x), \sigma_{A}(x), \gamma_{A}(x)>$

\section{Definition 3.6}

Let $\{A j: j \in J\}$ be a arbitrary family of NSS in $X$, then

(1) $\cap A j$ may be defined as two types:

(i) Type 1: $\cap A j=\left\langle x, \underset{j \in J}{\wedge} \mu_{A j}(x), \underset{j \in J}{\wedge} \sigma_{A j}(x), \vee \gamma_{A j}(x)\right\rangle$

(ii) Type 2: $\cap A j=\left\langle x, \wedge \mu_{A j}(x), \vee \sigma_{A j}(x), \vee \gamma_{A j}(x)\right\rangle$

(2) $\cup A j$ maybe defined as two types:

(i) Type1: $\cup A j=\langle x, \vee, \wedge, \wedge\rangle$

(ii) Type2: $\cup A j=\langle x, \vee, \wedge, \wedge\rangle$

\section{Definition 3.7}

Let $A$ and $B$ are neutrosophic sets then $A \mid B$ may be defined as

$A \mid B=\left\langle x, \mu_{A} \wedge \gamma_{B}, \sigma_{A}(x) \sigma_{B}(x), \gamma_{A} \vee \mu_{B}(x)\right\rangle$

\section{Proposition 3.2}

For all $A, B$ two neutrosophic sets then the following are true

(1) $C(A \cap B)=C(A) \cup C(B)$

(2) $C(A \cup B)=C(A) \cap C(B)$.

Salama et al. extended the concepts of fuzzy topological space [4], and intuitionistic fuzzy topological space [5, 7] to the case of neutrosophic sets.

\section{Definition 3.8}

A neutrosophic topology ( $N T$ for short) and a non empty set $X$ is a family $\tau$ of neutrosophic subsets in $X$ satisfying the following axioms

$$
\begin{aligned}
& \left(N T_{1}\right) O_{N}, 1_{N} \in \tau, \\
& \left(N T_{2}\right) G_{1} \cap G_{2} \in \tau \text { for any } \boldsymbol{G}_{1}, \boldsymbol{G}_{2} \in \tau, \\
& \left(N T_{3}\right) \cup \boldsymbol{G}_{\boldsymbol{i}} \in \tau \quad \forall\left\{\boldsymbol{G}_{\boldsymbol{i}}: \boldsymbol{i} \in \boldsymbol{J}\right\} \subseteq \tau
\end{aligned}
$$

In this case the pair $(X, \tau)$ is called a neutrosophic topological space (NTS for short) and any neutrosophic set in $\tau$ is known as neutrosophic open set (NOS for short) in $X$. The elements of $\tau$ are called open neutrosophic sets, A neutrosophic set $\mathrm{F}$ is closed if and only if it $\mathrm{C}(\mathrm{F})$ is neutrosophic open.

\section{Example 3.2}

Any fuzzy topological space $\left(X, \tau_{0}\right)$ in the sense of Chang is obviously a NTS in the form $\tau=\left\{A: \mu_{A} \in \tau_{0}\right\}$ wherever we identify a fuzzy set in $X$ whose members ship function is $\mu_{A}$ with its counterpart.

\section{Remark 3.3}

Neutrosophic topological spaces are very natural generalizations of fuzzy topological spaces allow more general functions to be members of fuzzy topology.

\section{Example 3.3}

Let $x=\{x\}$ and

$$
\begin{aligned}
A & =\{\langle x, 0.5,0.5,0.4\rangle: x \in X\} \\
B & =\{\langle x, 0.4,0.6,0.8\rangle: x \in X\} \\
D & =\{\langle x, 0.5,0.6,0.4\rangle: x \in X\} \\
C & =\{\langle x, 0.4,0.5,0.8\rangle: x \in X\}
\end{aligned}
$$

Then the family $\tau=\left\{O_{n}, 1_{n}, A, B, C, D\right\}$ of $\mathcal{N} S s$ in $X$ is neutrosophic topology on $X$.

\section{Example 3.4}

Let $\left(X, \tau_{0}\right)$ be a fuzzy topological space in changes sense such that $\tau_{0}$ is not indiscrete suppose now that $\tau_{0}=\left\{0_{N}, 1_{N}\right\} \cup\left\{V_{j}: j \in J\right\}$ then we can construct two NTSS on $X$ as follows
a) $\tau_{0}=\left\{0_{N}, 1_{N}\right\} \cup\left\{<x, V_{j}, \sigma(x), 0>: j \in J\right\}$.
b) $\tau_{0}=\left\{0_{N}, 1_{N}\right\} \cup\left\{<x, V_{j}, 0, \sigma(x), 1-V_{j}>: j \in J\right\}$.

\section{Proposition 3.3}

Let $(X, \tau)$ be an NTS on $X$, then we can also construct several NTSS on $X$ in the following way:
a) $\tau_{o, 1}=\{[] G: G \in \tau\}$,
b) $\tau_{o, 2}=\{\diamond G: G \in \tau\}$.

\section{Definition 3.9}


Let $\left(X, \tau_{1}\right),\left(X, \tau_{2}\right)$ be two neutrosophic topological spaces on $X$. Then $\tau_{1}$ is said be contained in $\tau_{2}$ (in symbols $\tau_{1} \subseteq \tau_{2}$ ) if $G \in \tau_{2}$ for each $G \in \tau_{1}$. In this case, we also say that $\tau_{1}$ is coarser than $\tau_{2}$.

\section{Proposition 3.4}

Let $\left\{\tau_{j}: j \in J\right\}$ be a family of NTSS on $X$. Then $\cap \tau_{j}$ is a neutrosophic topology on $X$. Furthermore, $\cap \tau_{j}$ is the coarsest $N T$ on $X$ containing all. $\tau_{j}, \mathrm{~s}$

\section{Definition 3.10}

The complement of $A$ (C (A) for short) of NOS. A is called a neutrosophic closed set ( NCS for short) in $X$.

Now, we define neutrosophic closure and interior operations in neutrosophic topological spaces:

\section{Definition 3.11}

Let $(X, \tau)$ be NTS and $A=<x, \mu_{A}(x), \gamma_{A}(x), \sigma_{A}(x)>$ be a $N S$ in $X$.

Then the neutrosophic closure and neutrosophic interior of Aare defined by

$$
\begin{gathered}
\operatorname{NCl}(A)=\cap\{K: K \text { is an NCS in } \mathrm{X} \text { and } \mathrm{A} \subseteq \mathrm{K}\} \\
\operatorname{NInt}(A)=\cup\{G: G \text { is an NOS in } \mathrm{X} \text { and } \mathrm{G} \subseteq \mathrm{A}\} .
\end{gathered}
$$

It can be also shown that $\operatorname{NCl}(A)$ is NCS and $\operatorname{NInt}(A)$ is a NOS in $X$

a) $A$ is in $X$ if and only if $\operatorname{NCl}(A)$.

b) $A$ is $\operatorname{NCSin} X$ if and only if $\operatorname{NInt}(A)=A$

\section{Proposition 3.5}

For any neutrosophic set $A$ in $(x, \tau)$ we have

(a) $\operatorname{NCl}\left(A^{c}\right)=(\operatorname{NInt}(A))^{c}$,

(b) $\operatorname{NInt}\left(A^{c}\right)=(N C L(A))^{c}$.

\section{Proposition 3.6}

Let $(X, \tau)$ be a NTS and $A, \mathrm{~B}$ be two neutrosophic sets in $X$. Then the following properties hold:
(a) $\operatorname{NInt}(A) \subseteq A$,
(b) $A \subseteq N C l(A)$,
(c) $A \subseteq B \Rightarrow \operatorname{NInt}(A) \subseteq \operatorname{NInt}(B)$
(d) $A \subseteq B \Rightarrow N C l(A) \subseteq N C l(B)$,
(e) $\operatorname{NInt}(\operatorname{NInt}(A))=\operatorname{NInt}(A)$
$N C L(N C L(A))=N C L(A)$
(f) $N C l(A \cup B)=N C l(A) \cup N C l(B)$,
$\operatorname{NInt}(A \cap B)=\operatorname{NInt}(A) \cap \operatorname{NInt}(B)$

(g) $\operatorname{NInt}\left(1_{N}\right)=1_{N}$,

(h) $\operatorname{NCl}\left(O_{N}\right)=O_{N}$

\section{SOME NeUtrosophic TOPOLOGICAL Notions OF NEUTROSOPHIC REGION}

In this section, we add some further definitions and propositions for a neutrosophic topological region.

\section{Corollary4.1}

Let $A=<\mu_{\mathrm{A}}(x), \sigma_{\mathrm{A}}(x), v_{A}(x)>\quad$ and $\quad B=<\mu_{B}(x), \sigma_{\mathrm{B}}(x), v_{B}(x)>$ are two neutrosophic sets on a neutrosophic topological space $(X, \tau)$ then the following are holds

i) $\quad N \operatorname{int}(A) \cap N \operatorname{int}(B)=N \operatorname{int}(A \cap B)$,

ii) $\operatorname{Ncl}(A) \cup \operatorname{Ncl}(B)=N \operatorname{int}(A \cup B)$,

iii) $N \operatorname{int}(A) \subseteq A \subseteq N c l(A)$,

iv) $(N \operatorname{int}(A))^{c}=N \operatorname{ccl}\left(A^{c}\right),(N c l(A))^{c}=N \operatorname{int}\left(A^{c}\right)$.

\section{Definition 4.1}

We define a neutrosophic boundary (NB) of a neutrosophic set $A=<\mu_{\mathrm{A}}(x), \sigma_{\mathrm{A}}(x), \nu_{A}(x)>$ by: $\partial A=\operatorname{Ncl}(A) \cap \operatorname{Ncl}\left(A^{c}\right)$.

The following theorem shows the intersection methods no longer guarantees a unique solution.

\section{Corollary 4.2:}

$\partial A \cap N \operatorname{int}(A)=O_{N} \quad$ iff $\quad N \operatorname{int}(A) \quad$ is $\quad$ crisp $\quad$ (i.e., $N \operatorname{int}(A)=O_{N}$ or $\left.N \operatorname{int}(A)=1_{N}\right)$.

Proof obvious

\section{Definition 4.2}

Let $A=<\mu_{\mathrm{A}}(x), \sigma_{\mathrm{A}}(x), v_{A}(x)>$ be a neutrosophic sets on a neutrosophic topological space $(X, \tau)$. Suppose that the family of neutrosophic open sets contained in A is indexed by the family $<\mu_{\mathrm{G}_{\mathrm{i}}}(x), \sigma_{\mathrm{G}_{\mathrm{i}}}(x), v_{G_{i}}(x)>: i \in I$ and the family of neutrosophic open subsets containing $\mathrm{A}$ are indexed the family $<\mu_{\mathrm{K}_{\mathrm{j}}}(x), \sigma_{\mathrm{K}_{\mathrm{j}}}(x), \nu_{K_{j}}(x)>: j \in J$.Then two neutrosophic interior, closure and boundaries are defined as following

a) $\left.\quad N \operatorname{int}(A)_{[}\right]$may be defined as two types

i) Type 1. $N \operatorname{int}(A)_{[]}=$ $<\max \left(\mu_{\mathrm{G}_{\mathrm{i}}}(x)\right), \max \left(\sigma_{\mathrm{G}_{\mathrm{i}}}(x)\right), \min \left(1-\mu_{G_{i}}(x)\right)>$

ii) Type 2. $N \operatorname{int}(A)_{[]}=$ $<\max \left(\mu_{\mathrm{G}_{\mathrm{i}}}(x)\right), \min \left(\sigma_{\mathrm{G}_{\mathrm{i}}}(x)\right), \min \left(1-\mu_{G_{i}}(x)\right)>$

b) $\quad N \operatorname{int}(A)_{<>}$may be defined as two types

i) Type 1. $N \operatorname{int}(A)_{<>}=$ $<\max \left(1-v_{\mathrm{G}_{\mathrm{i}}}(x)\right), \max \left(\sigma_{\mathrm{G}_{\mathrm{i}}}(x)\right), \min \left(v_{G_{i}}(x)\right)>$ 
ii. Type 2. $N \operatorname{int}(A)_{<>}$

$<\max \left(1-v_{\mathrm{G}_{\mathrm{i}}}(x)\right), \min \left(\sigma_{\mathrm{G}_{\mathrm{i}}}(x)\right), \min \left(v_{G_{i}}(x)\right)>$

c) $\left.\quad \operatorname{Ncl}(A)_{[}\right]$may be defined as two types

i) Type 1. $\operatorname{Ncl}(A)_{[]}=$

$<\max \left(\mu_{\mathrm{K}_{\mathrm{j}}}(x)\right), \min \left(\sigma_{\mathrm{K}_{\mathrm{j}}}(x)\right), \max \left(1-\mu_{K_{j}}(x)\right)>$

ii) Type 2. $\operatorname{Ncl}(A)_{[]}=$

$<\max \left(\mu_{\mathrm{K}_{\mathrm{j}}}(x)\right), \max \left(\sigma_{\mathrm{K}_{\mathrm{j}}}(x)\right), \max \left(1-\mu_{K_{j}}(x)\right)>$

d) $\quad \operatorname{Ncl}(A)_{<>}$may be defined as two types

i) Type 1. $\operatorname{Ncl}(A)_{<>}=$

$<\min \left(1-v_{\mathrm{K}_{\mathrm{i}}}(x)\right), \min \left(\sigma_{\mathrm{G}_{\mathrm{i}}}(x)\right), \max \left(v_{G_{i}}(x)\right)>$

ii) Type $2 \operatorname{Ncl}(A)_{<>}=$ $<\min \left(1-v_{\mathrm{K}_{\mathrm{i}}}(x)\right), \max \left(\sigma_{\mathrm{G}_{\mathrm{i}}}(x)\right), \max \left(v_{G_{i}}(x)\right)>$.

e) Neutrosophic boundaries defined as
i) $\left.\quad \partial A_{[]}=\operatorname{Ncl}\left(A_{[}\right]\right) \cap \operatorname{Ncl}\left(A_{[}^{c}{ }_{[}\right)$
ii) $\quad \partial A_{<>}=\operatorname{Ncl}\left(A_{<>}\right) \cap \operatorname{Ncl}\left(A^{c}<>\right)$

\section{Proposition 4.1}

a) $\left.N \operatorname{int}(A)_{[}\right] \subseteq N \operatorname{int}(A) \subseteq N \operatorname{int}(A)_{<>}$,

b) $\operatorname{Ncl}(A)_{[]} \subseteq \operatorname{Ncl}(A) \operatorname{Ncl}(A)_{<>}$

c) $\left.\left.N \operatorname{int}\left(A_{\{[}\right],<>\right\}\right)=\{[],<>\} N \operatorname{int}(A)$ and

$\left.\left.\operatorname{Ncl}\left(A_{\{[}\right],<>\right\}\right)=\{[],<>\} \operatorname{Ncl}(A)$

\section{Proof}

We shall only prove (c), and the others are obvious.

[ $] N \operatorname{int}(A)=<\max \left(\mu_{\mathrm{G}_{\mathrm{i}}}(x)\right), \max \left(\sigma_{\mathrm{G}_{\mathrm{i}}}(x)\right),\left(1-\max \mu_{G_{i}}(x)\right)>$ or $=<\max \left(\mu_{\mathrm{G}_{\mathrm{i}}}(x)\right), \min \left(\sigma_{\mathrm{G}_{\mathrm{i}}}(x)\right),\left(1-\max \mu_{G_{i}}(x)\right)>$

Based on knowing that $\left(1-\max \mu_{G_{i}}(x)\right)=\min \left(1-\mu_{G_{i}}\right)$ then [ ] $N \operatorname{int}(A)=<\max \left(\mu_{\mathrm{G}_{\mathrm{i}}}(x)\right), \max \left(\sigma_{\mathrm{G}_{\mathrm{i}}}(x)\right), \min \left(1-\mu_{G_{i}}(x)\right)>$ or $\quad<\max \left(\mu_{\mathrm{G}_{\mathrm{i}}}(x)\right), \min \left(\sigma_{\mathrm{G}_{\mathrm{i}}}(x)\right), \min \left(1-\mu_{G_{i}}(x)\right)>\quad=$

[ ] $N \operatorname{int}(A)$

In a similar way the others can prove.

\section{Proposition 4.2}

a) $\quad N \operatorname{int}\left(A_{\{[],<>\}}\right)=(N \operatorname{int}(A))_{\{[],<>\}}$

b) $\left.\left.\left.\left.\operatorname{Ncl}\left(A_{\{[}\right],<>\right\}\right)\{[],<>\}=(\operatorname{Ncl}(A))_{\{[}\right],<>\right\}$

\section{Proof}

Obvious

\section{Definition 4.3}

Let $A=<\mu_{\mathrm{A}}(x), \sigma_{\mathrm{A}}(x), v_{A}(x)>$ be a neutrosophic sets on a neutrosophic topological space $(X, \tau)$. We define neutrosophic exterior of A as follows: $A^{N E}=1_{N} \cap A^{C}$

\section{Definition 4.4}

Let $A=<\mu_{\mathrm{A}}(x), \sigma_{\mathrm{A}}(x), v_{A}(x)>$ be a neutrosophic open sets and $B=<\mu_{\mathrm{B}}(x), \sigma_{\mathrm{B}}(x), v_{B}(x)>$ be a neutrosophic set on a neutrosophic topological space $(X, \tau)$ then

a) $\mathrm{A}$ is called neutrosophic regular open iff $A=N \operatorname{int}(N \operatorname{cl}(A))$.

b) If $B \in N C S(X)$ then $\mathrm{B}$ is called neutrosophic regular closed iff $A=\operatorname{Ncl}(N \operatorname{int}(A))$.

Now, we shall obtain a formal model for simple spatial neutrosophic region based on neutrosophic connectedness.

\section{Definition 4.5}

Let $A=<\mu_{\mathrm{A}}(x), \sigma_{\mathrm{A}}(x), v_{A}(x)>$ be a neutrosophic sets on a neutrosophic topological space $(X, \tau)$. Then $\mathrm{A}$ is called a simple neutrosophic region in connected NTS, such that

i) $\left.\quad \operatorname{Ncl}(A), \operatorname{Ncl}(A)_{[}\right]$, and $\operatorname{Ncl}(A)_{<>}$are neutrosophic regular closed.

ii) $\quad N \operatorname{int}(A), N \operatorname{int}(A)_{[]}$, and $N \operatorname{int}(A)_{<>}$are neutrosophic regular open

iii) $\partial(A), \partial(A)_{[]}$, and $\partial(A)_{<>}$are neutrosophic connected.

Having $N \operatorname{cl}(A), N \operatorname{Ncl}(A)_{[]}, \quad N c l(A)_{<>}, N \operatorname{int}(A), N \operatorname{int}(A)_{[]}$, $N \operatorname{int}(A)_{<>}$are $\partial(A), \quad \partial(A)_{[]}$, and $\partial(A)_{<>}$for two neutrosophic regions, we enable to find relationships between two neutrosophic regions

\section{CONCLUSION}

Neutrosophic logic is well equipped to deal with missing data. By employing NSs in spatial data models, we can express a hesitation concerning the object of interest. This article has gone a step forward in developing methods that can be used to define neutrosophic spatial regions and their relationships. The main contributions of the paper can be described as the following: Possible applications have been listed after the definition of NS. Links to other models have been shown. We are defining some new operators to describe objects, describing a simple neutrosophic region. This paper has demonstrated that spatial object may profitably be addressed in terms of neutrosophic logic. Implementation of the named applications is necessary as a proof of concept.

\section{REFERENCES}

[1] K. Atanassov, intuitionistic fuzzy sets, in V.Sgurev, ed., Vii ITKRS Session, Sofia (June 1983 central Sci. and Techn. Library, Bulg. Academy of Sciences (1984).

[2] K. Atanassov, intuitionistic fuzzy sets, Fuzzy Sets and Systems 20, ,(1986),pp.87-96.

[3] K. Atanassov, Review and new result on intuitionistic fuzzy sets, preprint IM-MFAIS-1-88, Sofia, (1988).

[4] S. A. Alblowi, A.A. Salama and Mohmed Eisa, New 
Concepts of Neutrosophic Sets, International Journal of Mathematics and Computer Applications Research (IJMCAR),Vol. 4, Issue 1, (2014) ,pp.59-66.

[5] I.M. Hanafy, A.A. Salama and K. Mahfouz, Correlation of Neutrosophic Data, International Refereed Journal of Engineering and Science (IRJES), Vol.(1), Issue 2 .(2012), pp.39-33.

[6] J. F.Allen, "Maintaining Knowledge about Temporal Intervals." Communications of the ACM 26(11), (1983), pp.832-843.

[7] P. A. Burrough,. and A. U. Frank, Eds.. Geographic Objects wit Indeterminate Boundaries. GISDATA Series. London, Taylor \& Francis,(1996).

[8] T.Cheng, A Process-Oriented Data Model for Fuzzy Spatial Objects. Wageningen, Agricultural University,(1999).

[9] E. Clementini, E. and P. Di. Felice. An Algebraic Model for Spatial Objects with Indeterminate Boundaries. Geographic Objects with Indeterminate,(1996).

[10] Boundaries European Science Foundation. A. U. Frank, Taylor \& Francis. 2: pp.155- 169.

[11] E. Clementini, E. and P. Di Felice. "Approximate topological relations."International Journal of Approximate Reasoning 16,(1997), pp.173-204.

[12] A. G. Cohn, B. Bennet, et al. "RCC: a calculus for Region based Qualitative Spatial Reasoning." Geoinformatica 1,(1997), pp.275-316.

[13] A. G. Cohn, and N. M. Gotts. The 'egg-yolk' Representation of Regions with Indeterminate Boundaries. Geographic Objects with Indeterminate Boundaries. A. U. Frank. London, Taylor \& Francis. GISDATA II, (1996), pp.171-187.

[14] D. Coker, "A note on intuitionistic sets and intuitionistic points." TU .J. Math. 20(3),(1996) ,pp.343-351

[15] D. Coker,. "An introduction to intuitionistic fuzzy topological space." Fuzzy sets and Systems 88: (1997),pp. 81-89.

[16] M. J. Egenhofer, A Formal Definition of Binary Topological Relationships.Third International Conference on Foundations of Data Organization and Algorithms (FODO), Paris, France, June 1989, Springer- Verlag, Berlin Heidelberg, Germany (FRG).

[17] M. J. Egenhofer. and R. D. Franzosa ,"Point-set topological spatial relations." International Journal of Geographical Information Systems 5(2) ,(1996), pp.161-174.

[18] M. J. Egenhofer. and J. R. Herring. Categorizing Binary opological Relationships Between Regions, Lines, and Points in Geographic Databases., Department of Surveying Engineering, University of Maine, (1991).

[19] ME.Orono, P.Fisher. Boolean and fuzzy region. Geographic Objects with Indeterminate Boundaries. A. Frank, Taylor \& Francis:,(1996), pp.87-94.

[20] A. Gopnik, and A. N. Meltzoff. Words, Thoughts, and Theories.Cambridge, Mass., The MIT Press,(1997).

[21] M.N. Gotts, J. M. Gooday, et al.. "A connection based approach to common-sense topological description and reasoning." The Monist 79(1), (1995).

[22] M. Kokla,. and M. Kavouras. "Fusion of Top-level and Geographic Domain Omtologies based on Context Formation and Complementarity. "International Journal of Geographical Information Science 15(7),(2001), pp.679-687.

[23] F. Lehmann, and A. G. Cohn. The EGG/YOLK reliability data integration using sorts with prototypes. Infornmation Knowledge Management, ACM Press, (1994).

[24] M. Molenaar, An Introduction to the Theory of Spatial
Object Modelling for GIS. London, Taylor \& Francis,(1998).

[25] D.A.Randell. Z. Cui, et al. A spatial logic based on regions and conection. Third International Conference on the Principles of Knowledge Representation and Reasoning, Los Altos, CA: Morgan-Kaufmann,(1992).

[26] A.J.Roy.,A Comparison of Rough Sets, Fuzzy sets and Nonmonotonic Logic. Staffordshre, University of Keele,(1999).

[27] M. Schneider. Uncertainty Management for Spatial Data in Databases:Fuzzy Spatial Data Types. 6th Int. Symp. on Advances in Spatial, (1999).

[28] M.Schneider, "Finite Resolution Crisp and Fuzzy Spatial Objects". Int. Symp. on Spatial Data Handling, Databases, Springer-Verlag. (2000).

[29] M. Schneider, "A Design of Topological Predicates for Complex Crisp and Fuzzy Regions". Int. Conf. on Conceptual Modeling, (2001).

[30] M.Schneider, "Fuzzy Topological Predicates, Their Properties, and Their Integration into Query Languages". 9th ACM Symp. on Geographic Information Systems, (2001).

[31] J. G. Stell, and M. F. Worboys (1997). The Algebraic Structure of Sets of Regions. Spatial Information Theory (COSIT '97), Laurel Highlands, PA, Springer.

[32] X.Tang, and W. Kainz. "Analysis of Topological relations between Fuzzy Regions in a General Fuzzy Topological space". Symposium on Geospatial Theory, Processing and Applications, Ottawa,(2002).

[33] M. S .White, "A Survey of the Mathematics of Maps". Auto Carto IV,(1979).

[34] F. B. Zhan, "Approximate analysis of binary topological relations between geographic regions with indeterminate boundaries" Soft Computing 2,(1998), pp.28-34.

[35] I.M. Hanafy, A.A. Salama and K.M. Mahfouz,", Neutrosophic Classical Events and Its Probability" International Journal of Mathematics and Computer Applications Research(IJMCAR) Vol.(3),Issue 1,Mar (2013), pp.171-178.

[36] A. A. Salama and S.A. Alblowi, "Generalized Neutrosophic Set and Generalized Neutrosophic Spaces,"Journal Computer Sci. Engineering, Vol. (2) No. (7) (2012),pp.129-132 .

[37] A. A. Salama and S. A. Alblowi, "Neutrosophic Set and Neutrosophic Topological Spaces", ISOR J. Mathematics, Vol.(3), Issue(3), (2012) pp-31-35.

[38] A. A. Salama, "Neutrosophic Crisp Point \& Neutrosophic Crisp Ideals", Neutrosophic Sets and Systems, Vol.1, No. 1 , (2013) pp. 50-54.

[39] A. A. Salama and F. Smarandache, "Filters via Neutrosophic Crisp Sets", Neutrosophic Sets and Systems, Vol.1, No. 1, (2013) pp. 34-38.

[40] A. A. Salama and S.A. Alblowi, "Intuitionistic Fuzzy Ideals Spaces", Advances in Fuzzy Mathematics, Vol.(7), Number 1, (2012) pp. 51- 60.

[41] A.A. Salama, and H.Elagamy, "Neutrosophic Filters" International Journal of Computer Science Engineering and Information Technology Reseearch (IJCSEITR), Vol.3, Issue (1), Mar 2013,(2013), pp. 307-312.

[42] A. A. Salama, F.Smarandache and Valeri Kroumov "Neutrosophic crisp Sets \& Neutrosophic crisp Topological Spaces, Neutrosophic Sets and Systems, Vol.(2),(2014), pp.25-30.

[43] A. A. Salama, Mohamed Eisa and M. M Abdelmoghny,"Neutrosophic Relations Database" International Journal of Information Science and Intelligent System, 3(1), (2014). 
[44] A. A. Salama, F. Smarandache and S. A. ALblowi, "New Neutrosophic Crisp Topological Concepts", Neutrosophic Sets and Systems, Accsepted, (2014) .

[45] F. Smarandache, Neutrosophy and Neutrosophic Logic, First International Conference on Neutrosophy , Neutrosophic Logic, Set, Probability, and Statistics University of New Mexico, Gallup, NM 87301, USA, (2002).

[46] F. Smarandache, "A Unifying Field in Logics: Neutrosophic Logic. Neutrosophy, Neutrosophic crisp Set, Neutrosophic Probability. American Research Press, Rehoboth, NM, (1999).

[47] F. Smarandache, "Neutrosophic set, a generialization of the intuituionistics fuzzy sets", Inter. J. Pure Appl. Math., 24 (2005), $287-297$.

[48] D. Sarker, "Fuzzy ideal theory, Fuzzy local function and generated fuzzy topology", Fuzzy Sets and Systems 87, (1997),pp.117-123..

[49] L.A. Zadeh, "Fuzzy Sets", Inform and Control 8, 338-353.(1965).

[50] A. Salama, S. Broumi and F. Smarandache, Neutrosophic Crisp Open Set and Neutrosophic Crisp Continuity via Neutrosophic Crisp Ideals, IJ. Information Engineering and Electronic Business, Vol.6, No.3,(2014) 1-8.

\section{Authors' Profiles}

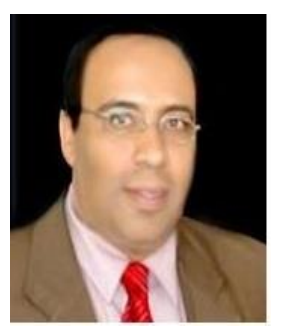

Dr.A.A. Salama (Ahmed Salama) Doctor and Lecturer in Mathematics and Computer Sciences Department in Faculty of Science in Port Said University. Associate Professor of Pure Mathematics \& Computer Science in Baha College of Sciences, Saudi Arabia. Obtained Doctoral degree in 2001 in Pure Mathematics. . He published over 100 articles and notes in mathematics, computer science and Statistics.

- The first Arab to use the Neutrosophic concepts in these areas (computer Sci., Math, Statistics and Topology).

- A member of its Editorial Board to International Journal's Neutrosophic Set and Systems (USA).

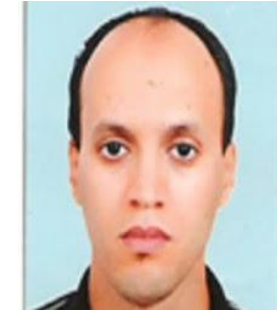

- He published over 100 articles and notes in mathematics, computer science and Statistics.

- Reviewers of The Book Marius Coman The Math Encyclopedia Of Smarandache Type Notions. I. Number Theory Educational Publishing, 2013 by Marius Coman Education Publishing USA.

- A member of Editorial Board SMARANDACHE NOTIONS Journal's Vol.iii, ii, i.USA.

- Manager of the Quality Assurance Unit, Port Said Faculty of Science.

- Head of the Committee of Training and Community Service, Al-Baha Private College of Science.

- Educational Supervisor of Mathematics in the Zahraa Islamic for Language Schools, Mansoura for six years.

- Secretary-general of Topology Conference held in the Suez Canal University, 2007.

- Staff Member in the Higher Institute Tebah for Computer and Administrative Sciences, Maadi, Cairo, Egypt.

- Head of the Board of Al-Haram Educational Periodical published in London.

- American Diploma Delegate, American Eagles Schools. 2005-2006.

- Main research points currently are Neutrosophic Mathematics, Computer Sciences and Statistics.

Said Broumi orked in Hassan II Casablanca university as an administrator. He worked in University for six years. He received his M. Sc in control system from Hassan II University Ain chok- Casablanca. A member of Editorial board to International Journal's Neutrosophic Set and Systems (USA).His research concentrates on soft set theory, fuzzy theory, intuitionistic fuzzy theory, neutrosophic theory, control systems. $\mathrm{He}$ has published 20 articles in international journals

Dr. Salwa Abdullah Rashed Alblowi: Doctor and Assistant professor in Mathematics Department in Faculty of Faculty of Sciences, King AbdulAziz University, Jeddah, Saudi Arabia.

How to cite this paper: A. A. Salama, Said Broumi, S. A. Alblowi,"Introduction to Neutrosophic Topological Spatial Region, Possible Application to GIS Topological Rules", IJIEEB, vol.6, no.6, pp.15-21, 2014. DOI: 10.5815/ijieeb.2014.06.02 